\title{
Miniaturized high-performance drift tube ion mobility spectrometer
}

\author{
André Ahrens $^{1}$ (D) - Moritz Hitzemann ${ }^{1} \cdot$ Stefan Zimmermann ${ }^{1}$
}

Received: 8 March 2019 / Revised: 26 March 2019 / Accepted: 28 March 2019/Published online: 4 April 2019

(C) The Author(s) 2019

\begin{abstract}
Developing powerful hand-held drift tube ion mobility spectrometers (IMS) requires small, lightweight drift tubes with high analytical performance. In this work, we present an easy-to-manufacture, miniaturized drift tube ion mobility spectrometer, which is manufactured from polyether ether ketone, stainless steel foils and printed circuit boards. It is possible to operate the drift tube IMS with a radioactive ${ }^{3} \mathrm{H}$ ionization source or a non-radioactive $\mathrm{X}$-ray ionization source with $3 \mathrm{kV}$ acceleration voltage. The drift tube design provides high resolving power of $R_{p}=63$ at a drift length of just $40 \mathrm{~mm}, 15 \mathrm{~mm} \times 15 \mathrm{~mm}$ in cross-section (outer dimensions) and a drift voltage of $2.5 \mathrm{kV}$. The limits of detection for less than one second of averaging are $40 \mathrm{ppt}_{\mathrm{v}}$ for dimethylmethylphosphonate and $30 \mathrm{ppt}_{\mathrm{v}}$ for methyl salicylate. For demonstration, the miniaturized drift tube IMS is integrated into a stand-alone battery-powered mobile device, including a closed gas-loop, high performance driver electronics and wireless data transmission. In a proof-of-concept study, this device was tested in an international field evaluation exercise to detect the release of a volatile, hazardous substance inside a large entry hall.
\end{abstract}

Keywords Miniaturized drift tube ion mobility spectrometer $\cdot$ Miniaturized drift tube IMS $\cdot$ Non-radioactive ionization $\cdot$ mini-IMS

\section{Introduction}

During the past decades, ion mobility spectrometers (IMS) have been established in various kinds of applications [1-4], while safety and security application still make up the largest share. Some IMS are used as stationary detectors for monitoring purpose; others are embedded into mobile or hand-held devices [5-7]. Especially in the latter case, compact, robust, lightweight and low cost drift tubes are required to provide easy to operate, hand-held devices. Thus, in recent years, several groups worked on the development of miniaturized drift tubes IMS. Some designs are based on low temperature cofired ceramic (LTCC) [8]. Other designs use stacked electrode-insulator-pairs $[6,9]$ or printed circuit boards $[10$, 11]. However, the key challenge of miniaturization is to maintain the analytical performance of the IMS, which strongly depends on the geometrical dimensions of the drift tube $[10$, 12].

André Ahrens

ahrens@geml.uni-hannover.de

1 Institute of Electrical Engineering and Measurement Technology, Department of Sensors and Measurement Technology, Leibniz University Hannover, Appelstr. 9A, 30167 Hannover, Germany
In drift tube IMS, ion separation is based on the motion of ions along the axis of the drift tube driven by a homogeneous electrical field. Two important performance indicators of IMS are the resolving power $\mathrm{R}_{\mathrm{p}}$ and the limits of detection (LoD). The resolving power, see Eq. 1, is defined as the quotient of the drift time $t_{d}$ and the full width at half maximum $\mathrm{w}_{\mathrm{FWHM}}$ of a peak in the spectrum.

$R_{p}=\frac{t_{d}}{w_{F W H M}}$

Typically, the LoD is defined as the concentration generating a signal amplitude equal to three times the standard deviation $\sigma$ of the noise at zero concentration. Furthermore, ion mobility is given as reduced ion mobility $\mathrm{K}_{0}$, see Eq. 2, where $\mathrm{L}$ is drift length, $\mathrm{U}_{\mathrm{d}}$ is drift voltage, $\mathrm{T}_{0}=273,15 \mathrm{~K}, \mathrm{p}_{0}=1013,25 \mathrm{mbar}$, $\mathrm{T}$ is operating temperature and $\mathrm{p}$ is operating pressure.

$K_{0}=\frac{L^{2}}{t_{d} \cdot U_{d}} \cdot \frac{T_{0}}{T} \cdot \frac{p}{p_{0}}$

In this work, we show a drift tube design, which is suitable for low cost and portable applications while maintaining the resolving power of laboratory-grade systems. The design is based on stainless steel straps, which are bended over a printed circuit board (PCB) and form the drift electrodes. Since the drift electrodes are connected to a dual-in-line package (DIP) 
footprint, the drift tube is easy to integrate simply being an electrical part of the driver electronics of the system.

\section{Miniaturized drift tube}

The basic layout of the drift tube presented in this work is similar to typical drift tube designs, consisting of an ionization region, an ion gate, a drift region and a shielded Faraday plate as ion detector. This basic drift tube setup and the corresponding operation principle have been known for years and are reported in detail elsewhere [13]. After being injected into the drift region, ions move along the axis of the drift tube driven by a homogeneous electrical field. During their motion, the ion species are separated based on their ion mobility in the counter-flow drift gas. At the end of the drift region, a faraday plate shielded by an aperture grid detects the ion current. By plotting this ion current over time, the ion mobility spectrum is obtained. Typically, the electric field in the drift tube is provided by several drift electrodes, which are connected via a resistive voltage divider to the drift voltage. To generate a homogeneous drift field, a precise alignment of all drift electrodes, insulators and further mechanical parts is essentially. Thus, when developing a miniaturized drift tube and aiming for low production cost, the total number of components should be small. For easy integration, the miniaturized drift tube design presented in this work is designed to fit commercially available DIP sockets ( $7.62 \mathrm{~mm}$ row-to-row spacing, 34 pins with $2.54 \mathrm{~mm}$ pitch). This enables direct connection of the drift tube to the driver electronic circuit. A standard PCB containing electrodes and etched gaps as well as arches of stainless steel straps bended over the PCB, as shown in Fig. 1a, form the drift electrodes. The straps are soldered to the bottom side of the PCB and connected by vias with the top layer electrodes to keep the drift region free from contaminants caused by the soldering process. The drift electrode skeleton is placed inside a U-shaped polyether ether ketone (PEEK) housing and is sealed at the bottom side with a onecompound epoxy resin adhesive, as shown in Fig. 1b. Since PEEK is a thermoplastic material, parts can be produced by low cost injection molding.

The miniaturized drift tube IMS is completed by an ionization chamber and a Faraday detector. The ionization chamber is also manufactured from PEEK and allows the adaption of two different ion sources to the drift tube. Ion generation is initiated either by electron emission from a radioactive ${ }^{3} \mathrm{H}$ source $(130 \mathrm{MBq})$ or by radiation from a custom nonradioactive X-ray source (Model XRT-50-2-Rh-0.6-125 by Newton Scientific Inc., Cambridge, Massachusetts, USA). It has been shown, that X-rays can be considered as nonradioactive substitute for the ${ }^{3} \mathrm{H}$ ionization source, but the $\mathrm{X}$ ray source should be placed orthogonally to the axis of the drift tube to avoid any offset current on the detector [14, 15]. Ions

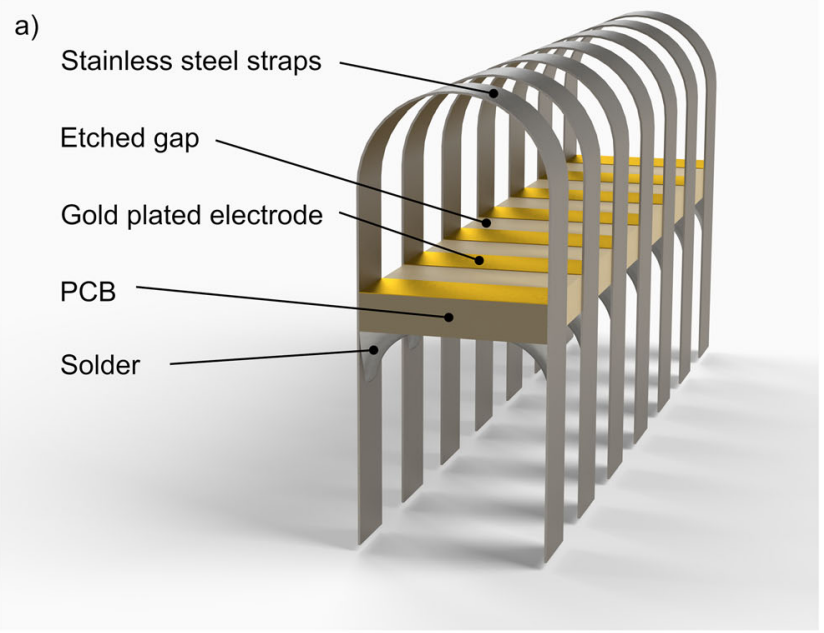

b)

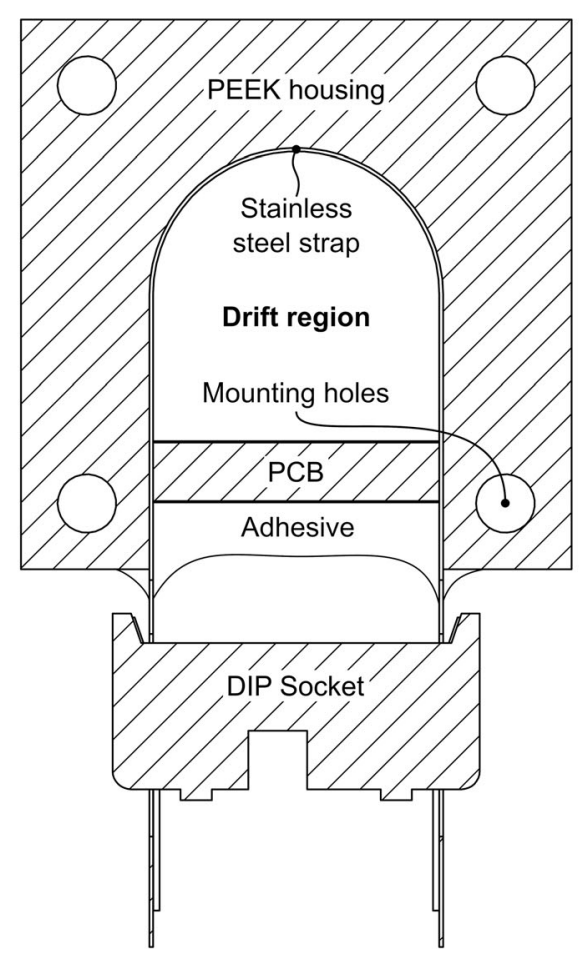

Fig. 1 (a) Exemplary design of the drift electrode skeleton formed by stainless steel straps and a PCB and (b) Section view of an assembled drift tube pluged into a DIP socket

are injected into the drift region using a field switching shutter as described in [16]. To keep production cost low, the Faraday detector is manufactured from standard PCBs. Ionization chamber, X-ray tube respectively, and Faraday detector are coupled to the drift tube using polytetrafluoroethylene (PTFE) O-rings and water jet cut foils.

For drift and sample gas inlet, ionization chamber and detector are equipped with bores fitting a PEEK capillary (1/16" outer diameter, $1 \mathrm{~mm}$ inner diameter). The sample gas is directly introduced into the ionization region, while the drift gas enters the drift tube at the detector through channels milled in the PCB. 
a)

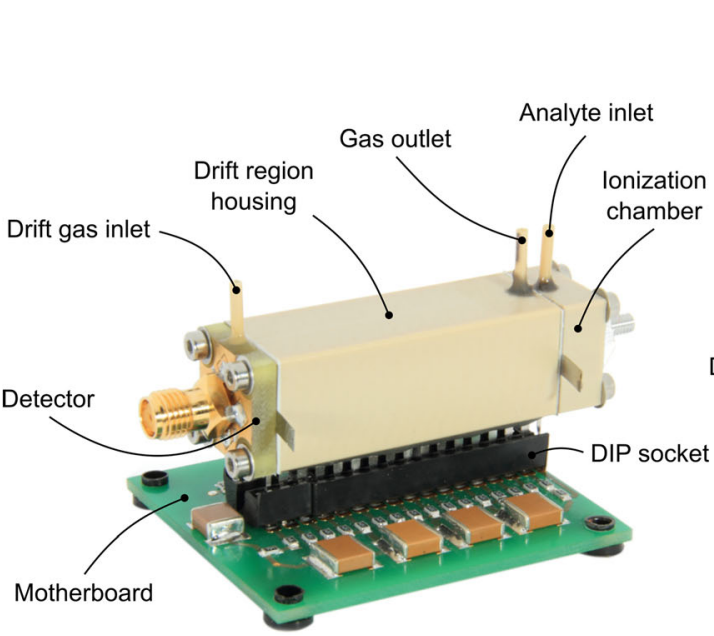

b)

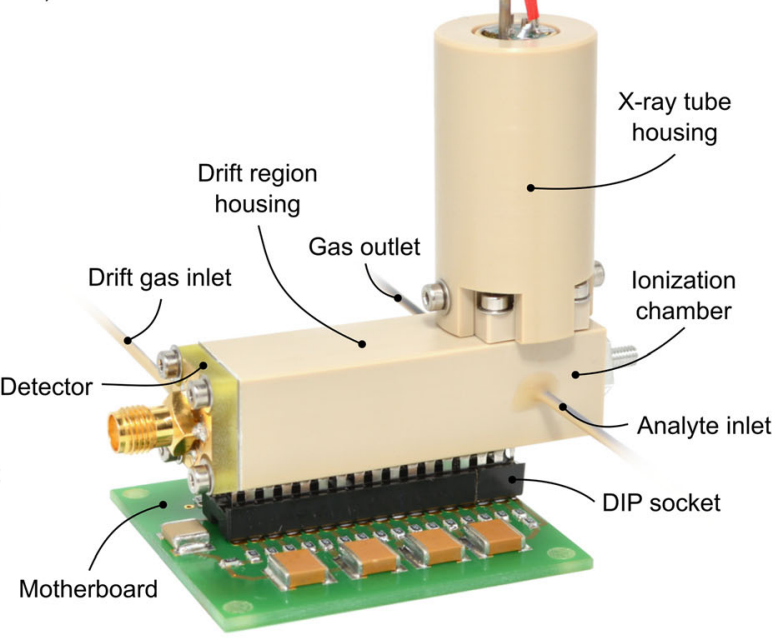

Fig. 2 Miniaturized drift tube IMS in a DIP socket on a motherboard with ${ }^{3} \mathrm{H}$ ionization source (a) and X-ray ionization source (b)

Fig. 3 Schematic of the internal gas flows within the stand-alone device

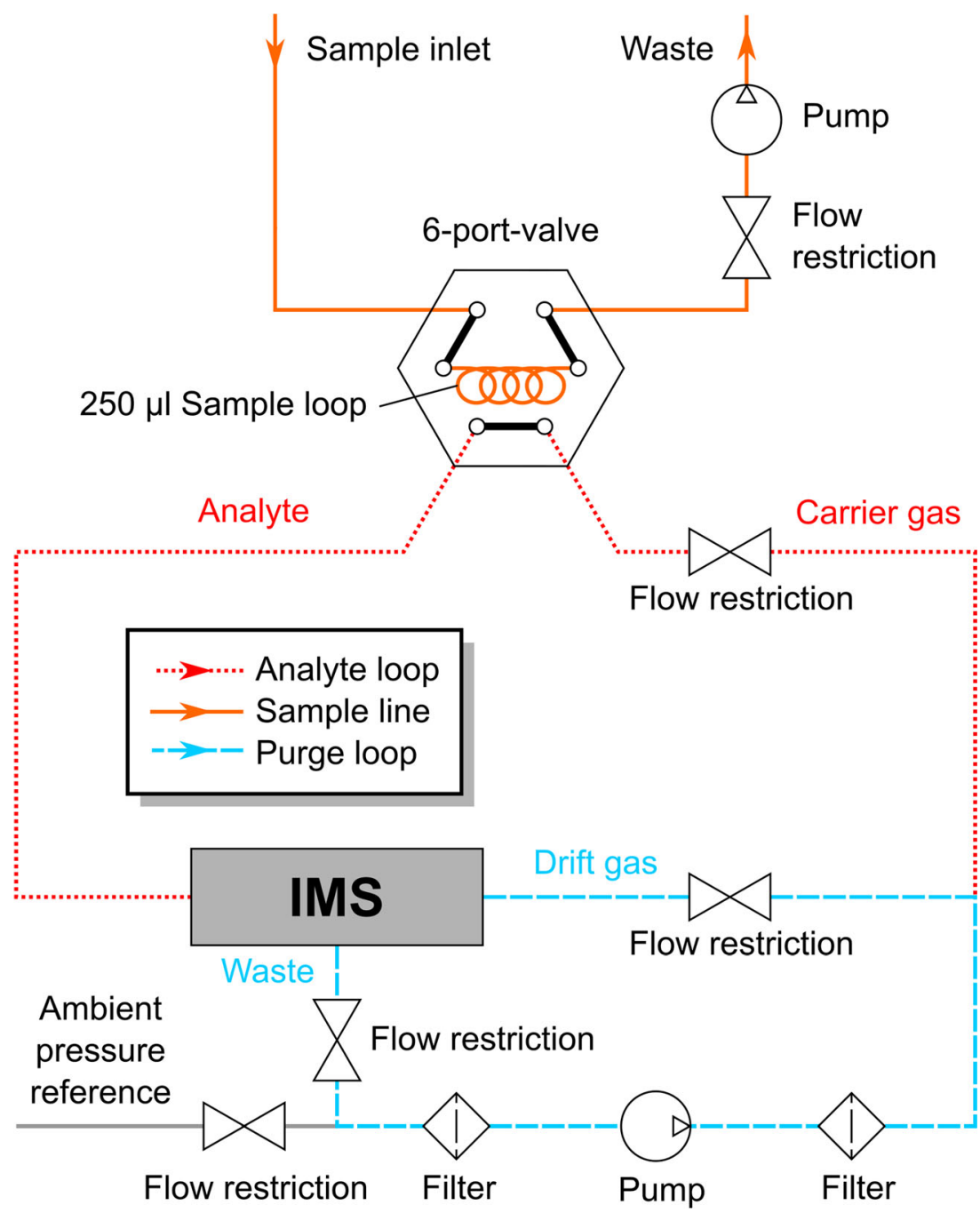


Fig. 4 Stand-alone demonstrator with closed gas-loop, direct inlet and miniaturized drift tube IMS with non-radioactive ionization source (and GC option for preseparation)

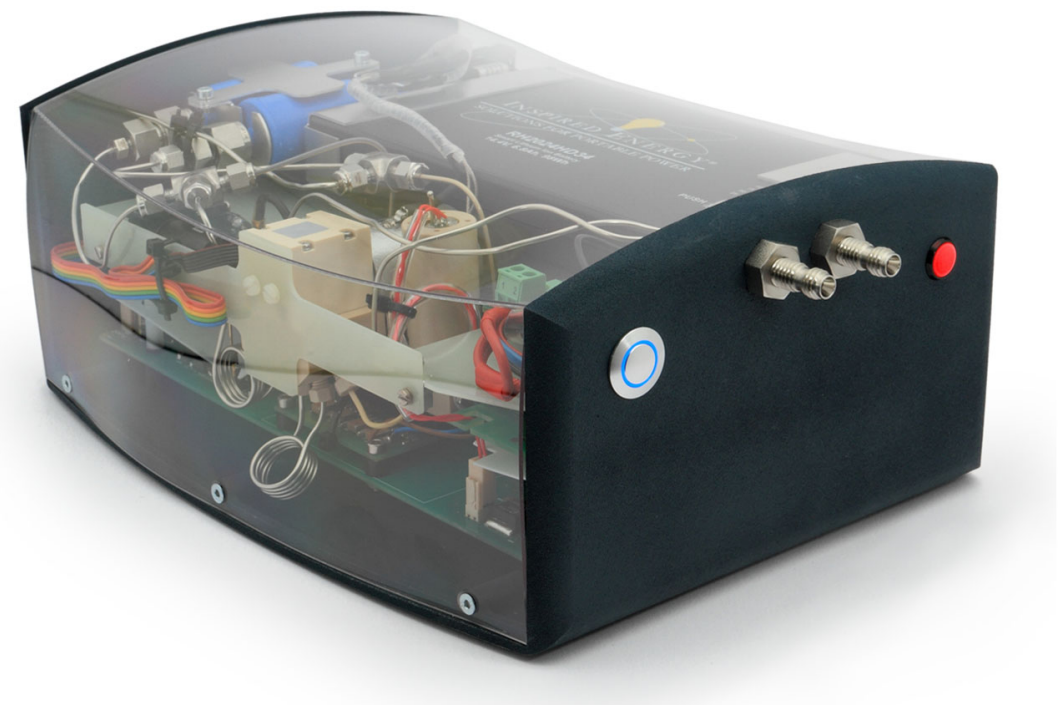

In Fig. 2, photos of the miniaturized drift tube with the ${ }^{3} \mathrm{H}$ ionization source (left) and the non-radioactive X-ray source (right) are shown. Both IMS are plugged into a DIP socket on a motherboard. The depicted housing of the drift tube is only $15 \mathrm{~mm} \times 15 \mathrm{~mm}$ in cross-section (outer dimensions). The overall length of the IMS including ionization chamber and detector is $56 \mathrm{~mm}$ (outer dimension) and the drift length is $40 \mathrm{~mm}$.

\section{Battery-powered mobile demonstrator with closed gas-loop}

To achieve high analytical power, a homogeneous drift field is crucial. Therefore, the drift tube is connected to a resistive voltage divider via the DIP socket, as described above. For all experiments, drift voltages in the range of $2.5 \mathrm{kV}$ are used. In addition, another high voltage of $3 \mathrm{kV}$ is required as

Table 1 Operating parameters of the IMS

\begin{tabular}{ll}
\hline Parameter & Value \\
\hline Drift length & $40 \mathrm{~mm}$ \\
Drift voltage & $2.5 \mathrm{kV}$ \\
Injection voltage & $500 \mathrm{~V}$ \\
X-ray tube filament current & $640 \mathrm{~mA}$ \\
X-ray tube acceleration voltage & $3.0 \mathrm{kV}$ \\
Repetition rate & $40 \mathrm{~Hz}$ \\
Drift gas flow & $120 \mathrm{ml} / \mathrm{min}$ \\
Sample gas flow & $100 \mathrm{ml} / \mathrm{min}$ \\
Analyte gas flow & $10 \mathrm{ml} / \mathrm{min}$ \\
Sample loop volume & $250 \mu \mathrm{l}$ \\
Inner IMS pressure & $1015 \mathrm{hPa}$ \\
IMS temperature & $25{ }^{\circ} \mathrm{C}$ \\
\hline
\end{tabular}

acceleration voltage for the X-ray source in the respective setup. The filament of the X-ray source is driven with a constant current of $640 \mathrm{~mA}$. The field-switching shutter is operated at injection voltages in the range of $500 \mathrm{~V}$.

The miniaturized drift tube IMS is integrated into a batterypowered mobile device including a closed gas-loop, high performance driver electronics, wireless data transmission and on-board data storage. To avoid contamination and provide clean and dry drift gas, the air inside the closed gas-loop is continuously pumped through two $13 \mathrm{X}$ type disposable filters (Balston Filter No. 9922-05-103 by Parker Hannifin Corporation, Haverhill, Massachusetts, USA). Figure 3 illustrates a schematic view of the pneumatic set up. Sample gas $\left(100 \mathrm{ml}_{\mathrm{s}} / \mathrm{min}\right)$, drift gas $\left(120 \mathrm{ml}_{\mathrm{s}} / \mathrm{min}\right)$ and carrier gas $\left(10 \mathrm{ml}_{\mathrm{s}} /\right.$ min) flow rates are controlled by the pumping rates and passive flow restrictions. The demonstrator including the lithium ion battery, (14.4 V, $6.8 \mathrm{Ah}$, part No. RH2024HD34 by Inspired Energy) and electronics (in-house development), pumps (models NMP05KPDC-B4 and NMP015KPDC-B4 by KNF Neuberger GmbH, Freiburg, Germany), filters, amplifier (in-house development, $900 \mathrm{M} \Omega$ transimpedance, $16 \mathrm{kHz}$ bandwidth), 6-port-valve (Model MTV-6SL-N32UF1 by Takasago Electric Inc., Nagoya, Japan) is built in a custom $25.5 \mathrm{~cm} \times 20.5 \mathrm{~cm} \times 10.8 \mathrm{~cm}$ housing and has a total weight of about $2 \mathrm{~kg}$. The demonstrator is shown in Fig. 4.

A defined sample gas flow of $100 \mathrm{ml}_{\mathrm{s}} / \mathrm{min}$ (at $20{ }^{\circ} \mathrm{C}$ and $1013.25 \mathrm{hPa}$ ) is periodically pumped from the sample inlet (Fig. 3 - orange, continuous line) through the $250 \mu$ sample loop. During measurements, the sample loop pump is turned off to avoid any interferences caused by mechanical vibrations, while the closed gas-loop pump is continuously running. When a measurement is started, the gas within the sample loop is injected via 6-port-valve into the IMS by a carrier gas flow of $10 \mathrm{ml}_{\mathrm{s}} / \mathrm{min}$ (Fig. 3 - red, dotted line). The 6-port-valve also allows easy integration 
Fig. 5 (a) Positive reactant ion peak $\left(\mathrm{RIP}^{+}, \mathrm{K}_{0}=2.01 \mathrm{~cm}^{2} /(\mathrm{Vs})\right)$ and (b) negative reactant ion peaks $\left(\mathrm{RIP}^{-} \mathrm{K}_{0}=2.15 \mathrm{~cm}^{2} /(\mathrm{Vs})\right.$ and $\left.\mathrm{K}_{0}=2.08 \mathrm{~cm}^{2} /(\mathrm{Vs})\right)$, both in purified, dry air

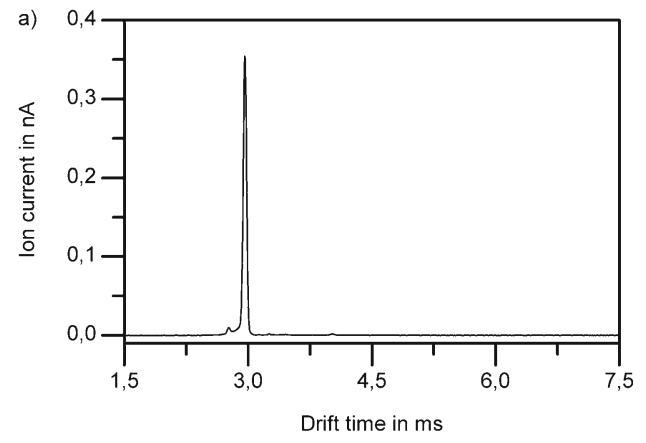

of a gas chromatographic column for pre-separation (GC-IMS). An ambient pressure reference (Fig. 3 - grey, continuous line) is added before the filters to avoid unwanted pressure variations inside the closed gas-loop e.g. during valve switching.

All operating parameters are summarized in Table 1. The IMS can be operated in positive and negative ion mode.

\section{Results and discussion}

To analyze the performance of the presented IMS, we measured its resolving power and detection limits. In a first step, the miniaturized drift tube is characterized without being integrated in the stand-alone demonstrator. Afterwards, the standalone demonstrator is investigated.

\section{Analytical performance of the miniaturized drift tube}

In Fig. 5, the ion mobility spectrum of purified, dry $\left(<1 \mathrm{ppm}_{\mathrm{V}}\right.$ $\mathrm{H}_{2} \mathrm{O}$ ) air is shown, both in positive and negative ion mode. The resolving power of the positive reactant ion peak $\left(\mathrm{RIP}^{+}\right)$at $t_{d}=2.96 \mathrm{~ms}$ is $R_{p}=63$, while the resolving power of the negative reactant ion peak $\left(\right.$ RIP $\left.^{-}\right)$at $t_{d}=2.74 \mathrm{~ms}$ is $R_{p}=62$. The spectra are measured using the ${ }^{3} \mathrm{H}$ ionization source.

In order to determine the detection limit and linear dynamic response range of the drift tube IMS, dimethylmethylphosphonate (DMMP) has been used as model substance in the positive ion mode and methyl salicylate (MS) has been used as model substance in the negative ion mode. In this work, all limits of detection are given for measurement times less than one second and are based on the $3 \sigma$-definition.
In Fig. 6, the ion mobility spectrum of $1.5 \mathrm{ppb}_{\mathrm{V}}$ DMMP in purified, dry air with its monomer at $t_{d}=3.35 \mathrm{~ms}$ and dimer at $\mathrm{t}_{\mathrm{d}}=4.27 \mathrm{~ms}$ is shown. Using the ${ }^{3} \mathrm{H}$-ionization source, a detection limit of $40 \mathrm{ppt}_{\mathrm{V}}$ is calculated considering the monomer peak, respectively $500 \mathrm{ppt}_{\mathrm{V}}$ considering the dimer peak. Using the X-ray ionization source, the detection limits the DMMP monomer and dimer are about $60 \mathrm{ppt}_{\mathrm{V}}$ and $800 \mathrm{ppt}_{\mathrm{V}}$. It is important to note, that an increasing X-ray filament current leads to significantly better limits of detection. However, here, the operating parameters of the used X-ray ionization source were set to generate RIP intensities comparable to ${ }^{3} \mathrm{H}$ ionization. To characterize the negative ion mode, MS is used. The detection limits of MS measured with ${ }^{3} \mathrm{H}$-ionization source, is calculated to $30 \mathrm{ppt}_{\mathrm{V}}$ and with $\mathrm{X}$-ray ionization is calculated to $60 \mathrm{ppt}_{\mathrm{V}}$. The $\mathrm{K}_{0}$ value for MS is $1.55 \mathrm{~cm}^{2} /(\mathrm{Vs})$.

\section{Analytical performance of the stand-alone demonstrator}

As described in the previous section, the miniaturized drift tube IMS with X-ray ionization has been integrated into a mobile demonstrator. Using the closed gas-loop with 6-port-valve sample injection results in a detection limit of $90 \mathrm{ppt}_{\mathrm{V}}$ for MS in purified, dry air. Compared to the detection limit using the drift tube IMS without closed gas-loop and 6-port-valve sample injection, this value is higher by a factor of three. This is due to dilution of the sample when mixing with the carrier gas.

As proof-of-concept study, the stand-alone device was tested in an international field evaluation exercise of European Union's TOXI-triage project in Athens in October 2018. The device was used as stationary detector to detect the release of a
Fig. 6 Ion mobility spectrum of $1.5 \mathrm{ppb}_{\mathrm{V}} \mathrm{DMMP}\left(\mathrm{K}_{0}=1.76 \mathrm{~cm}^{2} /\right.$ (Vs) (monomer), $\mathrm{K}_{0}=1.38 \mathrm{~cm}^{2} /$ (Vs) (dimer) in clean, dry air $\left({ }^{3} \mathrm{H}\right.$ ionization source (a) and X-ray ionization (b))
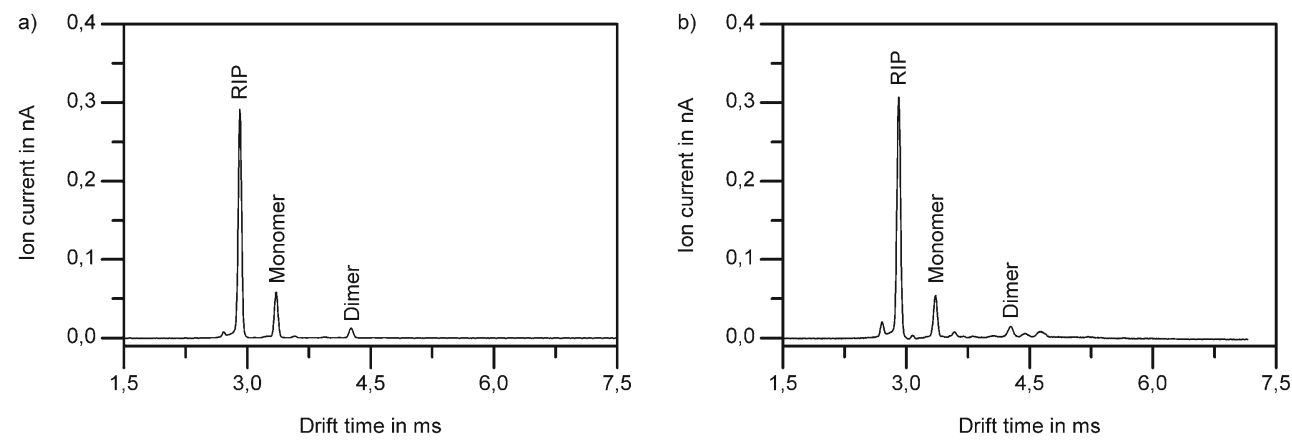

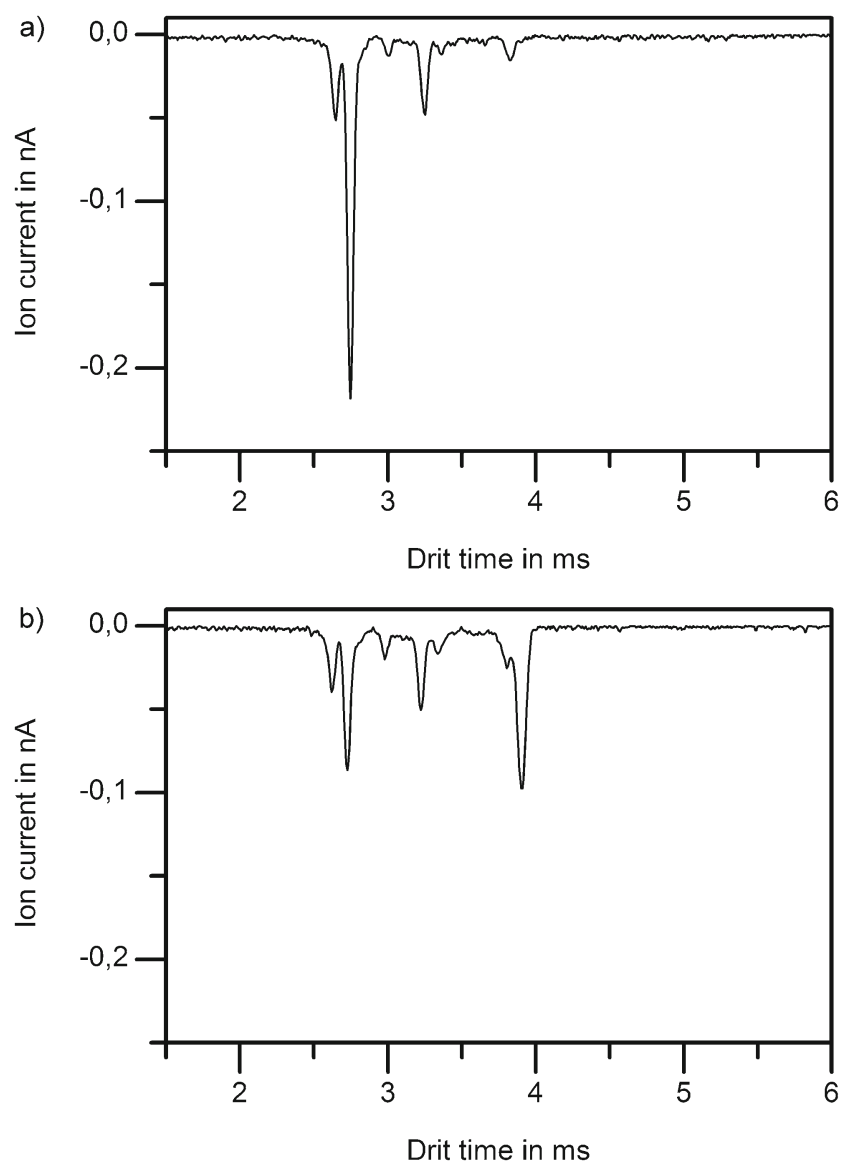

Fig. 7 Ion mobility spectra taken at TOXI-triage's field evaluation exercise: (a) ambient air; (b) simulant release

volatile, hazardous substance inside a large entry hall. Here, a non-hazardous compound simulated this substance. Due to the high amount of compounds in the ambient air and a direct sample inlet without pre-separation, the resulting IMS spectrum is rather complex, as shown in Fig. 7 (top). Nonetheless, it is possible to detect the simulant substance. Additionally, in Fig. 7, the ion mobility spectrum of the air in the hall at release time (Fig. 7 - bottom) of the simulant $\left(t_{d}=3.91 \mathrm{~ms}\right)$ is given.

\section{Conclusion}

In this work, an easy-to-manufacture, miniaturized drift tube ion mobility spectrometer is presented. The design is based on stainless steel straps, which are bended over a printed circuit board (PCB) and form the drift electrodes. Since the drift electrodes are connected to a dual-in-line package (DIP) footprint, the drift tube is easy to connect to the driver electronics (plug and play).

The dimensions of the drift tube are only $15 \mathrm{~mm} \times 15 \mathrm{~mm}$ in cross-section (outer dimensions) and $56 \mathrm{~mm}$ in length (outer dimension). Nevertheless, the design provides a high resolving power of $R_{p}=63$ at a drift length of just $40 \mathrm{~mm}$ and a drift voltage of $2.5 \mathrm{kV}$. The limits of detection for one second of averaging are $40 \mathrm{ppt}_{\mathrm{v}}$ for dimethyl-methylphosphonate monomer, $500 \mathrm{ppt}_{\mathrm{V}}$ for the dimer and $30 \mathrm{ppt}_{\mathrm{v}}$ for methyl salicylate using a ${ }^{3} \mathrm{H}$ ionization source. Using X-ray ionization, the limits of detection are $60 \mathrm{ppt}_{\mathrm{v}}$ for dimethylmethylphosphonate monomer, $800 \mathrm{ppt}_{\mathrm{V}}$ for the dimer and $60 \mathrm{ppt}_{\mathrm{v}}$ for methyl salicylate. It is important to note, that an increasing X-ray filament current leads to significantly better limits of detection. However, here, the operating parameters of the used X-ray ionization source were set to generate RIP intensities comparable to ${ }^{3} \mathrm{H}$ ionization. The system has been successfully tested in an international field evaluation exercise to detect the release of volatile, hazardous material inside a large entry hall.

Acknowledgements This project has received funding from the European Union's Horizon 2020 research and innovation programme under grant agreement No 653409.

\section{Compliance with ethical standards}

Conflict of interest The authors declare that they have no conflict of interest.

Open Access This article is distributed under the terms of the Creative Commons Attribution 4.0 International License (http:// creativecommons.org/licenses/by/4.0/), which permits unrestricted use, distribution, and reproduction in any medium, provided you give appropriate credit to the original author(s) and the source, provide a link to the Creative Commons license, and indicate if changes were made.

\section{References}

1. Karpas Z (2013) Applications of ion mobility spectrometry (IMS) in the field of foodomics. Food Res Int 54:1146-1151

2. Perl T, Carstens E, Hirn A, Quintel M, Vautz W, Nolte J, Jünger M (2009) Determination of serum propofol concentrations by breath analysis using ion mobility spectrometry. Br J Anaesth 103:822827

3. Allers M, Langejuergen J, Gaida A, Holz O, Schuchardt S, Hohlfeld JM, Zimmermann S (2016) Measurement of exhaled volatile organic compounds from patients with chronic obstructive pulmonary disease (COPD) using closed gas loop GC-IMS and GC-APCI-MS. J Breath Res 10:26004

4. Eiceman GA, Stone JA (2004) Peer reviewed: ion mobility spectrometers in National Defense. Anal Chem 76:390A-397A

5. Snyder AP, Harden CS, Brittain AH, Kim MG, Arnold NS, Meuzelaar HLC (1993) Portable hand-held gas chromatography/ ion mobility spectrometry device. Anal Chem 65:299-306

6. Babis JS, Sperline RP, Knight AK, Jones DA, Gresham CA, Denton MB (2009) Performance evaluation of a miniature ion mobility spectrometer drift cell for application in hand-held explosives detection ion mobility spectrometers. Anal Bioanal Chem 395(2): 411-419

7. Eiceman GA, Snyder AP, Blyth DA (1990) Monitoring of airborne organic vapors using ion mobility spectrometry. Int J Environ Anal Chem 38:415-425 
8. Pfeifer KB, Rohde SB, Peterson KA, Rumpf AN (2004) Development of rolled miniature drift tubes using low temperature co- fired ceramics (LTCC). Int J Ion Mobil Spec 7

9. Pfeifer KB, Sanchez RC (2002) Miniaturized ion mobility spectrometer system for explosives and contraband detection. Int. J. Ion Mobil. Spec. 5:63-66

10. Bohnhorst A, Kirk AT, Zimmermann S (2016) Simulation aided design of a low cost ion mobility spectrometer based on printed circuit boards. Int J Ion Mobil Spec 19:167-174

11. Reinecke T, Clowers BH (2018) Implementation of a flexible, open-source platform for ion mobility spectrometry. HardwareX 4:e00030

12. Kirk AT, Allers M, Cochems P, Langejuergen J, Zimmermann S (2013) A compact high resolution ion mobility spectrometer for fast trace gas analysis. Analyst 138:5200-5207

13. Eiceman GA, Karpas Z, Hill HH (2013) Ion mobility spectrometry, 3rd edn. CRC Press, Boca Raton
14. Reinecke T, Kirk AT, Heptner A, Niebuhr D, Bottger S, Zimmermann S (2016) A compact high-resolution X-ray ion mobility spectrometer. Rev Sci Instrum 87(5):53120

15. Bunert E, Reinecke T, Kirk AT, Bohnhorst A, Zimmermann S (2018) Ion mobility spectrometer with orthogonal X-ray source for increased sensitivity. Talanta 185:537-541

16. Kirk AT, Zimmermann S (2014) Bradbury-Nielsen vs. field switching shutters for high resolution drift tube ion mobility spectrometers. Int. J. Ion Mobil. Spec. 17:131-137

Publisher's note Springer Nature remains neutral with regard to jurisdictional claims in published maps and institutional affiliations. 